\title{
Optical and infrared observations of the X-ray source 1WGA J1713.4-3949 in the G347.3-0.5 SNR ${ }^{\star}$
}

\author{
R. P. Mignani ${ }^{1}$, S. Zaggia ${ }^{2}$, A. De Luca ${ }^{3}$, R. Perna ${ }^{4}$, N. Bassan ${ }^{3}$, and P. A. Caraveo ${ }^{3}$ \\ 1 Mullard Space Science Laboratory, University College London, Holmbury St. Mary, Dorking, Surrey, RH5 6NT, UK \\ e-mail: rm2@mssl.ucl.ac.uk \\ 2 INAF - Osservatorio Astronomico di Padova, Vicolo dell'Osservatorio 5, Padua 35122, Italy \\ e-mail: simone.zaggia@oapd.inaf.it \\ 3 INAF - Istituto di Astrofisica Spaziale, via Bassini 15, Milan 20133, Italy \\ e-mail: [deluca; bassan;pat]@iasf-milano.inaf.it \\ 4 JILA and Department of Astrophysical and Planetary Sciences, University of Colorado, 440 UCB, Boulder, 80309, USA \\ e-mail: rosalba@jilau1.Colorado.EDU
}

Received 16 November 2007 / Accepted 17 March 2008

\section{ABSTRACT}

\begin{abstract}
Context. X-ray observations unveiled the existence of enigmatic point-like sources at the centre of young supernova remnants (SNRs). These sources, dubbed central compact objects (CCOs), are thought to be neutron stars formed by the supernova explosion. However, their multi-wavelength phenomenology is surprisingly different from that of most young neutron stars.

Aims. The aim of this work is to understand the nature of the CCO 1WGA J1713.4-3949 in the G347.3-0.5 SNR through deep optical and infrared (IR) observations, the first ever performed for this source.

Methods. By exploiting its derived Chandra X-ray position we carried out optical (BVI) observations with the New Technology Telescope (NTT) and adaptive optics IR $\left(J H K_{\mathrm{s}}\right)$ observations with the Very Large Telescope (VLT).

Results. We detected two faint $(I \approx 23.5, I \approx 24.3$.) patchy objects close to the Chandra error circle in the NTT images. They were clearly resolved in our VLT images which unveiled a total of six candidate counterparts $(17.8<H<20.3)$ with quite red colours $\left(H-K_{\mathrm{s}} \sim 0.6\right)$. If they are stars, none of them can be associated with 1WGA J1713.4-3949 for the most likely values of distance and hydrogen column density. The identification of the faintest candidate with the neutron star itself can not be firmly excluded, while the identification with a fallback disk is ruled out by its non-detection in the $I$ band. No other candidates are detected down to $B \sim 26$, $V \sim 26.2, I \sim 24.7, H \sim 21.3$, and $K \sim 20.5$.

Conclusions. Our high-resolution IR imaging unveiled a few objects close/within the Chandra X-ray position of 1WGA J1713.4-3949. However, at present none of them can be firmly identified as the object's likely counterpart.
\end{abstract}

Key words. stars: neutron - stars: individual: 1WGA J1713.4-3949

\section{Introduction}

X-ray observations have unveiled the existence of peculiar classes of isolated neutron stars (INSs). These INSs stand apart from the family of more classical radio pulsars in being radiosilent and not powered by the neutron star rotation but by still poorly understood emission mechanisms. Some of the most puzzling classes of radio-silent INSs are identified with a group of $\mathrm{X}$-ray sources detected at the centre of young ( $\sim 10-40$ kyears) supernova remnants (SNRs), hence dubbed central compact objects or CCOs (Pavlov et al. 2002).

Although the SNR associations imply ages of the order of a few kyears, their X-ray properties make CCOs completely different from the other young INSs in SNRs (Pavlov et al. 2004; De Luca 2008). Only two of them exhibit X-ray pulsations, with periods in the $\sim 100-400 \mathrm{~ms}$ range, and the measured upper limits on the period derivatives yield spin down ages $\geq 10^{3}$ exceeding the SNR age. Furthermore, their X-ray spectra are not purely magnetospheric but have strong thermal components. Finally, they are not embedded in pulsar wind nebulae (PWN). The

* Based on observations collected at the European Southern Observatory (ESO), La Silla and Paranal, Chile under programme ID 073.D-0632(A), 077.D-0764(A). discovery of long-term, X-ray flux variations (Gotthelf et al. 1999) and of a $6.7 \mathrm{~h}$ periodicity (e.g., De Luca et al. 2006) in the RCW 103 CCO further complicated the picture, suggesting either a binary system with a low-mass companion or a longperiod magnetar (De Luca et al. 2006; Pizzolato et al. 2008). For other CCOs, the invoked scenarios involve low-magnetised INSs surrounded by debris disks formed after the supernova event (Gotthelf \& Halpern 2007; Halpern et al. 2007), isolated accreting black holes (Pavlov et al. 2000), and dormant magnetars (Krause et al. 2005). In the optical/IR, deep observations have been performed only for a handful of objects (see De Luca 2008 , for a summary) but no counterpart has been identified yet, with the possible exception of the Vela Jr. CCO (Mignani et al. 2007a).

One of the CCOs that still lacks a deep optical/IR investigation is 1WGA J1713.4-3949 in the young ( $\leq 40$ kyears) G347.3-0.5 SNR. The source was discovered with Röntgen Satellite (ROSAT) by Pfeffermann \& Aschenbach (1996) and re-observed with the Advanced Satellite for Cosmology and Astrophysics (ASCA). It was identified as an INS due to its high-temperature spectrum and the lack of an optical counterpart (Slane et al. 1999). The source was later observed with the Rossi X-ray Timing Explorer (RXTE), Chandra, and the 
X-ray Multi-Mirror (XMM) satellites (Lazendic et al. 2003; Cassam-Chenaï et al. 2004). All of these observations were consistent with steady $\mathrm{X}$-ray emission. The X-ray luminosity is $L_{0.5-10 \mathrm{keV}} \sim 6 \times 10^{34}(\mathrm{~d} / 6 \mathrm{kpc})^{2} \mathrm{erg} \mathrm{s}^{-1}$, where $6 \mathrm{kpc}$ is the originally estimated SNR distance (Slane et al. 1999). A revised distance of $1.3 \pm 0.4 \mathrm{kpc}$ was recently obtained by Cassam-Chenaï et al. (2004). The X-ray spectrum can be fitted either by a blackbody, likely produced from hot polar caps, plus a power-law ( $k T \sim 0.4 \mathrm{keV} ; \Gamma \sim 4 ; N_{\mathrm{H}} \sim 9 \times 10^{21} \mathrm{~cm}^{-2}$ ), or by two blackbodies $\left(k T_{1} \sim 0.5 \mathrm{keV} ; k T_{2} \sim 0.3 \mathrm{keV} ; N_{\mathrm{H}} \sim 5 \times 10^{21} \mathrm{~cm}^{-2}\right)$. No Xray pulsations were detected so far (Slane et al. 1999; Lazendic et al. 2003), nor any radio counterpart (Lazendic et al. 2004), thus strengthening the case for 1WGA J1713.4-3949 as a member of the CCO class.

Here we present the results of the first, deep optical/IR observations of 1WGA J1713.4-3949 performed with the European Southern Observatory (ESO) telescopes. Observations are described in Sect. 2, while the results are described and discussed in Sects. 3 and 4, respectively.

\section{Observations and data reduction}

\subsection{Optical observations}

We observed 1WGA J1713.4-3949 on June 13, 2004 at the ESO La Silla Observatory with the New Technology Telescope (NTT). The telescope was equipped with the second generation of the SUperb Seeing Imager (SUSI2). The camera is a mosaic of two $2000 \times 4000$ pixels EEV CCDs with a $2 \times 2$ binned pixel scale of 0 .'16 (5.5 $\times 5$ 5.5 field of view $)$.

We obtained repeated exposures in the broad-band $B, V$, and $I$ filters. The SUSI2 observations $\log$ is summarised in the first half of Table 1. Observations were performed with the target close to the zenith and under reasonably good seeing conditions $\left(\sim 1^{\prime \prime}\right)$. Since the target was always centred on the left chip, no dithering was applied to the $B$ and $V$-band exposures while the $I$-band ones were dithered to compensate for the fringing pattern affecting the CCD at longer wavelengths. We acquired both night (twilight flat-fields) and daytime calibration frames (bias, dome flat-fields). Unfortunately, due to the presence of clouds both at the beginning and at the end of the night no standard star observations were acquired. As a reference for the photometric calibration we used the zero points closest in time to our observations, regularly measured using Landolt stars (Landolt 1983) as part of the instrument calibration plan, and available in the photometry calibration database maintained by the NTT/SUSI2 team. According to the zero point trending plots ${ }^{1}$, we estimate a conservative uncertainty of $\sim 0.1 \mathrm{mag}$ on the values extrapolated to the night of our observations.

\subsection{Infrared observations}

We observed 1WGA J1713.4-3949 on May 23 and 24, 2006 at the ESO Paranal Observatory with NAos COnica (NACO), the adaptive optics (AO) imager and spectrometer mounted at the Very Large Telescope (VLT) Yepun telescope. In order to provide the best combination between angular resolution and sensitivity, we used the S27 camera with a pixel scale of 0 . $^{\prime} 027$ (28" $\times$ $28^{\prime \prime}$ field of view). As a reference for the AO correction we used the GSC-2 star S230012111058 ( $V=14.3)$, positioned 11'.5

\footnotetext{
1 http://wwW.1s.eso.org/lasilla/sciops/ntt/susi/docs/ susiCounts.html
}

Table 1. Log of the NTT/SUSI2 (first half) and VLT/NACO (second half) observations of the 1WGA J1713.4-3949 field. Columns report the observing epoch, the filter, the total integration time, and the average seeing and airmass.

\begin{tabular}{ccccc}
\hline \hline yyyy-mm-dd & Filter & $T(\mathrm{~s})$ & Seeing $\left(^{\prime \prime}\right)$ & Airmass \\
\hline $2004-06-13$ & $B$ & 3200 & 1.14 & 1.07 \\
$2004-06-13$ & $V$ & 6400 & 1.12 & 1.03 \\
$2004-06-13$ & $I$ & 3150 & 1.0 & 1.16 \\
\hline $2006-05-24$ & $K_{\mathrm{s}}$ & 1800 & 0.66 & 1.45 \\
$2006-05-24$ & $K_{\mathrm{s}}$ & 360 & 0.95 & 1.70 \\
$2006-05-24$ & $K_{\mathrm{s}}$ & 600 & 0.78 & 1.81 \\
$2006-05-25$ & $H$ & 2400 & 0.62 & 1.42 \\
$2006-05-25$ & $K_{\mathrm{s}}$ & 1200 & 0.40 & 1.74 \\
\hline
\end{tabular}

away from our target, with the visual (4500-10000 A) dichroic element and wavefront sensor.

We observed in the $H$ and $K_{\mathrm{s}}$ bands. To allow for subtraction of the variable IR sky background, we split each integration in sequences of short randomly dithered exposures with detector integration times (DIT) of $24 \mathrm{~s}$ and 5 exposures (NDIT) along each node of the dithering pattern. The NACO observations $\log$ is summarised in the second half of Table 1. For all observations, the seeing conditions were on average below $\sim 0{ }^{\prime} 8$. Unfortunately, since we always observed the target at the end of the night, the airmass was always above 1.4. Sky conditions were photometric in both nights. On the first night, the second and third $K_{\mathrm{s}}$-band exposure sequence were aborted because the very high airmass prevented a correct reference star acquisition. Because of their worse image quality and their much lower signal-to-noise, these data are not considered in the following analysis. The $K_{\mathrm{s}}$-band exposure sequence obtained on the second night was interrupted despite of very good seeing because of the incoming twilight. Thanks to the combination of good seeing and low airmass, the $H$-band exposure has the best image quality. Night (twilight flat-fields) and daytime calibration frames (darks, lamp flat-fields) were taken daily as part of the NACO calibration plan. Standard stars from the Persson et al. (1998) fields were observed on both nights for photometric calibration.

\subsection{Data reduction}

We reduced the NTT/SUSI2 data using standard routine available in the MIDAS data reduction package ${ }^{2}$. After the basic reduction steps (hot pixels masking, removal of bad CCD column, bias subtraction, flat-field correction), single science frames were combined to filter cosmic ray hits and to remove the fringing patterns in the $I$-band. We computed the astrometry using the coordinates and positions of 61 stars selected from the 2MASS catalogue (Skrutskie et al. 2006). For a better comparison with the VLT/NACO IR images, the I-band image was taken as a reference. The pixel coordinates of the $2 M A S S$ stars (all non saturated and evenly distributed in the field) were measured by fitting their intensity profiles with a Gaussian function using the Graphical Astronomy and Image Analysis (GAIA) tool ${ }^{3}$. The fit to celestial coordinates was computed using the Starlink package ASTROM ${ }^{4}$. The rms of the astrometric fit residuals was $\approx 0$ ' 09 per coordinate. After accounting for the 0.2 conservative astrometric

\footnotetext{
2 http://www . eso.org/sci/data-processing/software/ esomidas/

3 star-www.dur.ac.uk/ pdraper/gaia/gaia.html

4 http://star-www.rl.ac.uk/Software/software.htm
} 

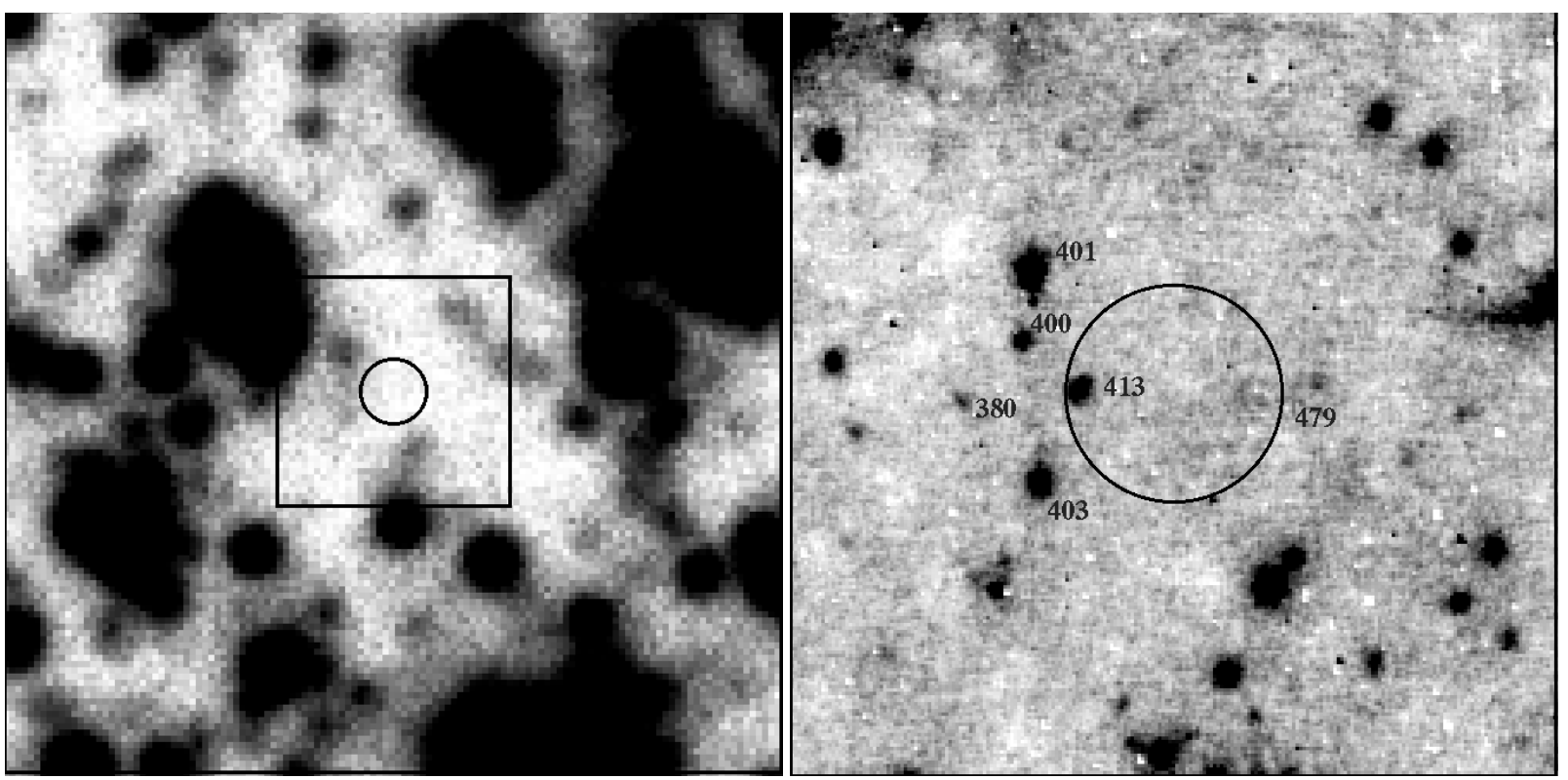

Fig. 1. (left) $20^{\prime \prime} \times 20^{\prime \prime}$ NTT/SUSI2 I-band of the 1WGA J1713.4-3949 field. (right) 6" $\times 6^{\prime \prime}$ VLT/NACO H-band image of the same field. The area corresponds to the square overplotted on the left-hand side image. The circle marks the Chandra position of the X-ray source, where the radius (0.'85) accounts both for the intrinsic absolute accuracy of the Chandra coordinates (99\% confidence level) and for the uncertainties of our astrometric calibration (see Sect. 2.3). Possible counterparts are labelled on the right-hand side image, with object 479 detected only at $<5 \sigma$.

accuracy of 2 MASS (Skrutskie et al. 2006), the overall uncertainty to be attached to our astrometry is finally 0.24 .

We processed the VLT data through the ESO NACO data reduction pipeline ${ }^{5}$. For each band, science frames were reduced with the produced master dark and flat-field frames and combined to correct for the exposure dithering and to produce cosmic-ray free and sky-subtracted images. The photometric calibration was applied using the zero point provided by the $N A C O$ pipeline, computed through fixed aperture photometry. The astrometric calibration was performed using the same procedure described above. However, since only five $2 M A S S$ stars are identified in the narrow NACO S27 camera field of view, we computed the astrometric solution using as a reference a set of 23 secondary stars found in common with the SUSI2 I-band image, calibrated using $2 M A S S$. The rms of the astrometric fit residuals was then $\approx 0$.'06 per coordinate. By adding in quadrature the rms of the astrometric fit residuals of the SUSI2 I-band image and the average astrometric accuracy of $2 M A S S$ we thus end up with an overall accuracy of 0.25 on the NACO image astrometry.

\section{Data analysis and results}

\subsection{Astrometry}

We derived the coordinates of 1WGA J1713.4-3949 through the analysis of unpublished Chandra observations. The field of 1WGA J1713.4-3949 was observed on April 19, 2005 with the ACIS/I instrument for $9.7 \mathrm{ks}$. Calibrated (level 2) data were retrieved from the Chandra X-ray Center Archive and were analysed using the Chandra Interactive Analysis of Observations software (CIAO v3.3). In order to compute the target position, we performed a source detection in the $0.5-10 \mathrm{keV}$ energy range using the wavdetect task. The source coordinates turned out to be $\alpha(J 2000)=17^{\mathrm{h}} 13^{\mathrm{m}} 28.32^{\mathrm{s}}$, $\delta(J 2000)=-39^{\circ} 49^{\prime} 53^{\prime \prime}$. 34, with a nominal uncertainty of $\sim 0^{\prime} .^{\prime} 8$

\footnotetext{
5 www.eso.org/observing/dfo/quality/NACO/pipeline
}

(99\% confidence level) ${ }^{6}$. The identification of a field X-ray source with the bright star HD 322941 at a position consistent with the one listed in the Tycho Reference Catalog (Høg et al. 2000) confirmed the accuracy of the nominal Chandra astrometric solution. Unfortunately, since no other field X-ray source could be unambiguously identified with catalogued objects, it was not possible to perform any boresight correction to the Chandra data to improve the nominal astrometric accuracy.

The computed 1WGA J1713.4-3949 position is shown in Fig. 1, overplotted on the NTT/SUSI2 I-band and on the VLT/NACO $\mathrm{H}$-band images. In Fig. 1 (left) a faint and patchy object is clearly detected northeast of the Chandra error circle $(I=23.5 \pm 0.3)$ and a fainter one $(I=24.3 \pm 0.4)$ is possibly detected south of it. However, in both cases, their patchy structure makes it difficult to determine whether they are single or blended with unresolved field objects. No other object is detected within or close to the Chandra error circle down to $B \sim 26, V \sim 26.2$, and $I \sim 24.7(3 \sigma)$. However, due to the better seeing conditions (see Table 1) and to the sharper angular resolution, five objects are clearly detected in the VLT/NACO image (Fig. 1-right). Of these, object 413 falls within the Chandra error circle. A sixth, fainter object (479) is possibly detected, albeit at very low significance. They are all point-like and compatible with the onaxis $N A C O$ point spread function (PSF). Objects 401 and 403 are identified with the two faint objects detected in the NTT/SUSI2 $I$-band image northeast and southeast of the Chandra error circle, respectively. The former might be actually a blend of objects 401 and 400 , whose angular separation $\left(\approx 0{ }^{\prime} 6\right)$ is smaller than the PSF of the NTT/SUSI2 image. We thus view the measured magnitude $(I=23.5 \pm 0.3)$ of object 401 with caution. All the objects detected in the $N A C O H$-band image are also detected in the longest 1200 and $1800 \mathrm{~s} K_{\mathrm{s}}$-band images (see Table 1 ). No other object is detected close to the Chandra error circle down to $H \sim 21.3$ and $K_{\mathrm{s}} \sim 20.5(3 \sigma)$.

\footnotetext{
${ }^{6}$ http://cxc.harvard. edu/cal/ASPECT/celmon/
} 
Table 2. VLT/NACO $H$ and $K_{\mathrm{s}}$-band photometry and colour of the candidate counterparts of 1WGA J1713.4-3949.

\begin{tabular}{cccc}
\hline \hline ID & $H$ & $K_{\mathrm{s}}$ & $H-K_{\mathrm{s}}$ \\
\hline 380 & $19.75 \pm 0.14$ & $19.32 \pm 0.11$ & $0.43 \pm 0.18$ \\
400 & $18.98 \pm 0.13$ & $18.49 \pm 0.09$ & $0.49 \pm 0.16$ \\
401 & $17.82 \pm 0.13$ & $17.32 \pm 0.08$ & $0.50 \pm 0.15$ \\
403 & $18.47 \pm 0.13$ & $17.87 \pm 0.08$ & $0.60 \pm 0.15$ \\
413 & $18.63 \pm 0.13$ & $18.31 \pm 0.09$ & $0.33 \pm 0.16$ \\
479 & $20.34 \pm 0.15$ & $19.61 \pm 0.12$ & $0.73 \pm 0.20$ \\
\hline
\end{tabular}

\subsection{Photometry}

We computed the magnitudes of the objects in the NACO images through PSF photometry using the suite of tools Daophot(Stetson 1992) and applying the same procedures described in Zaggia et al. (1997) and applied in Mignani et al. (2007a) and De Luca et al. (2008). Since the NACO PSF is largely oversampled, we re-sampled the images with a $3 \times$ 3 pixels window using the swarp program ${ }^{7}$ to increase the signal-to-noise ratio. As a reference for our photometry we used the co-added and re-sampled $H$-band image to create a master list of objects, which we registered on the $K_{\mathrm{s}}$-band one and used as a mask for the object detection. For each image, the model PSF was calculated by fitting the profile of a number of bright, but non-saturated reference stars in the field and used to measure the objects fluxes at the reference positions. Our photometry was calibrated using the zero points provided by the $N A C O$ pipeline after applying the aperture correction, with attached errors of $\sim 0.13$ and $\sim 0.08 \mathrm{mag}$ in $H$ and $K_{\mathrm{s}}$, respectively. We then matched single band catalogues and used them as a reference for our colour analysis. The IR magnitudes of our candidates are listed in Table 2 . We used the $K_{\mathrm{s}}$-band photometry performed on the two consecutive nights to search for variability on timescales of hours. However, none of our candidates show flux variations larger than $0.1 \mathrm{mag}$, which is consistent with our photometric errors. Figure 2 shows the $H, H-K_{\mathrm{s}}$ colour magnitude diagram (CMD) for our candidates as well as for all objects detected in the field. None of the candidates is characterised by peculiar colours with respect to the main sequence of the field stellar population, which suggest that they are main-sequence stars.

\section{Discussion}

To determine whether one of the detected objects is the IR counterpart to 1WGA J1713.4-3949, we investigated how their observed properties fit with different scenarios.

\subsection{A binary system}

If our candidates are stars, we considered the possibility that one of them is the companion of the 1WGA J1713.4-3949 neutron star. Their observed colours are quite red $\left(0.4<H-K_{\mathrm{s}}<\right.$ 0.7 , which suggests that they might be intrinsically red latetype stars. To be compatible with the observed range of $H-K_{\mathrm{s}}$ (Ducati et al. 2001), e.g. an M-type main sequence star should be reddened by an amount of interstellar extinction corresponding to an $N_{\mathrm{H}} \sim 10^{22} \mathrm{~cm}^{-2}$ (Predhel \& Schmitt 1995). This value is compatible with the largest values obtained from the spectral fits to 1WGA J1713.4-3949 (Lazendic et al. 2003; Cassam-Chenaï et al. 2004). For the originally proposed 1WGA J1713.4-3949

\footnotetext{
7 http://terapix.iap.fr/
}

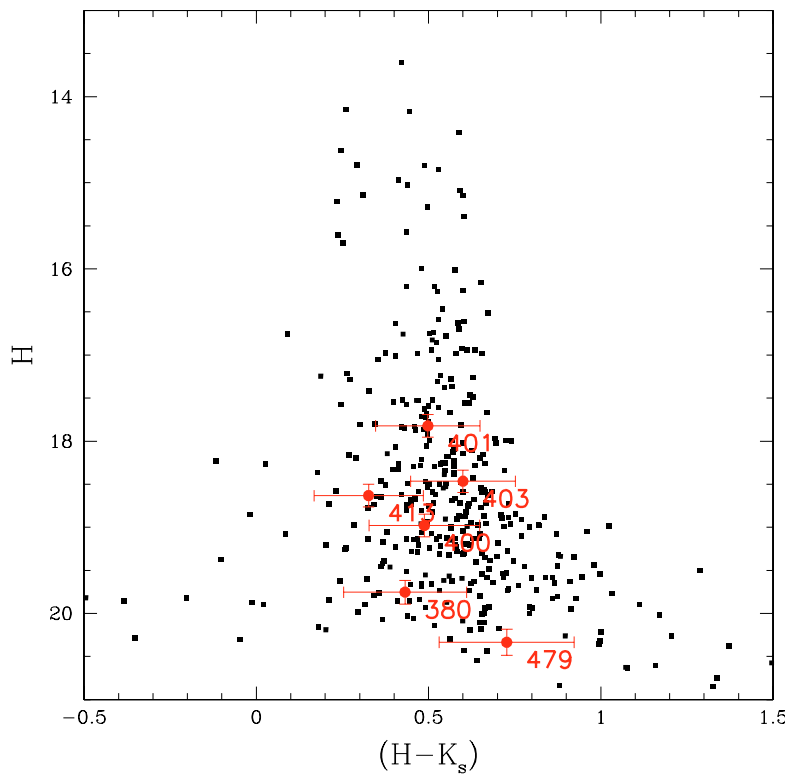

Fig. 2. $H, H-K_{\mathrm{s}} \mathrm{CMD}$ of all stars detected in the VLT/NACO field. All candidates identified in Fig. 1 are marked in red and labelled accordingly. No interstellar extinction correction has been applied.

distance of $6 \mathrm{kpc}$ (Slane et al. 1999) an M-type star with such an high extinction should be at least $\sim 0.7$ mag fainter than our faintest candidate (object 479). An early to mid M-type star would be compatible with the revised distance of $1.3 \pm 0.4 \mathrm{kpc}$ (Cassam-Chenaï et al. 2004), but it would be detected in our NTT/SUSI2 image at $I \sim 20.2-21.7$. Thus we conclude that, if our candidates are stars, none of them can be associated with 1WGA J1713.4-3949. Our optical/IR magnitude upper limits only allow an undetected companion of spectral type later than M.

\subsection{An isolated neutron star}

If 1WGA J1713.4-3949 is indeed an INS, we can then speculate whether one of our candidates is the neutron star itself. Due to the paucity of the neutron stars observed in the IR (e.g., Mignani et al. 2007b) and due to the lack of well-defined spectral templates, it is very difficult to estimate their expected IR brightness. This is even more difficult for CCO neutron stars, none of which has been unambiguosuly identified so far (e.g., De Luca 2008). In the best characterised case of rotation-powered neutron stars one can deduce that the magnetospheric IR and X-ray luminosities correlate (Mignani et al. 2007b; Possenti et al. 2002). By assuming, e.g., a blackbody plus power-law, X-ray spectrum for 1WGA J1713.4-3949 (Lazendic et al. 2003; Cassam-Chenaï et al. 2004), we then scaled the magnetospheric IR-to-X-ray luminosity ratio of the Vela pulsar, taken as a reference because of its comparable age ( $\sim 10$ kyears $)$. After accounting for the corresponding interstellar extinction, we thus estimated $K_{\mathrm{s}} \sim 19.7$ for 1WGA J1713.4-3949, i.e. similar to the magnitude of object $479\left(K_{\mathrm{s}} \sim 19.6\right)$. Also, since the magnetospheric optical and X-ray luminosities correlate (e.g., Zharikov et al. 2004), we similarly estimated $B \approx 28.3$ for 1WGA J1713.4-3949. This, however, is below our NTT/SUSI2 upper limit $(B \geq 26)$. Thus, a neutron star identification can not be firmly excluded. 


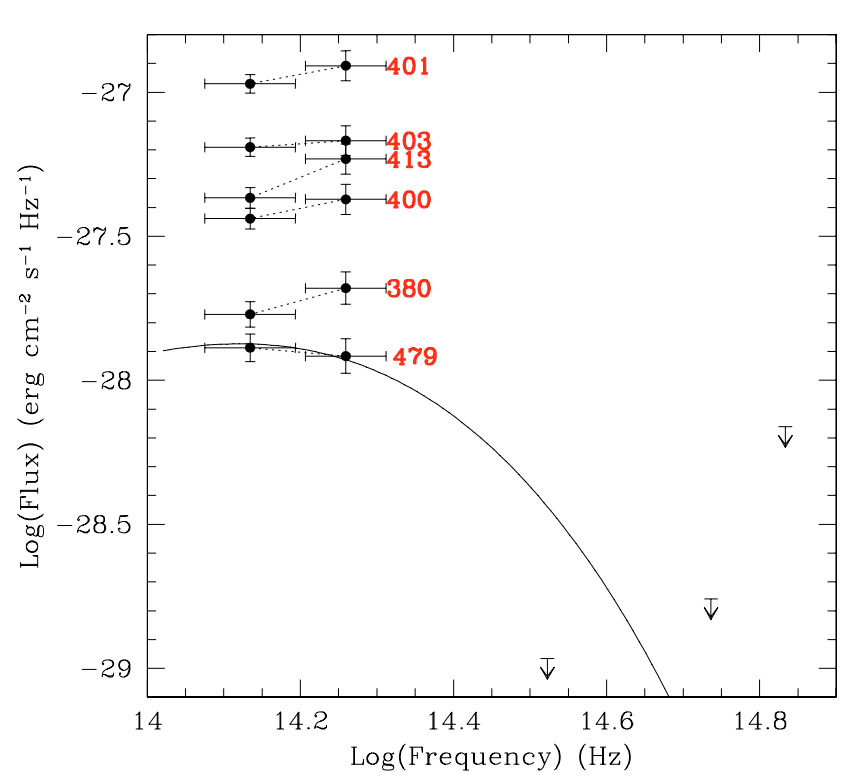

Fig. 3. Dereddened IR spectra of the 1WGA J1713.4-3949 candidate counterparts. Dotted lines are drawn for guidance. The $B V I$ bands upper limits are indicated. The solid line is the best fitting disk spectrum $\left(R_{\text {in }}=\right.$ $\left.0.28 R_{\odot}, R_{\text {out }}=1.4 R_{\odot}\right)$ for object 479 .

\subsection{A fossil disk}

As discussed in Sect. 1, some CCO models invoke lowmagnetised INSs surrounded by fallback disks. So, the last possibility is that we detected the IR emission from such a disk. We note that the IR-to-X-ray flux ratio for 1WGA J1713.4-3949 would be $\approx 10^{-3}-10^{-2}$, i.e. much larger than that estimated for the anomalous X-ray pulsar 4U 0142+61 (Wang et al. 2006), the only INS with evidence of a fallback disk. However, we can not a priori rule out the fallback disk scenario. We computed the putative disk emission using the model of Perna et al. (2000), which accounts for both for the contribution of viscous dissipation as well as that due to reprocessing of the neutron star X-ray luminosity. As a reference, we assumed the X-ray luminosity derived for the updated distance of $1.3 \pm 0.4 \mathrm{kpc}$ (Cassam-Chenaï et al. 2004). For a nominal disk inclination angle of $60^{\circ}$ with respect to the line of sight, the unknown model parameters are the disk inner and outer radii $\left(R_{\text {in }}, R_{\text {out }}\right)$ and the accretion rate $(\dot{M})$. We thus iteratively fitted our data for different sets of the model parameters. For the dimmest candidate we found that the IR fluxes would be consistent with a spectrum of a disk $\left(R_{\text {in }}=0.28 R_{\odot}\right.$, $R_{\text {out }}=1.4 R_{\odot}$ ) whose emission is dominated by the reprocessed neutron star X-ray luminosity (Fig. 3), similar to the case of $4 \mathrm{U} 0142+61$. However, such a disk should be detected in the $I$ band, with a flux $\sim 1.5$ mag above our measured upper limit, as shown in Fig. 3. The overprediction of the optical flux is even more dramatic for a disk that fits the brighter counterparts. We conclude that if the neutron star has a disk, it was not detected by our observations.

\section{Conclusions}

We performed deep optical and IR observations of the CCO 1WGA J1713.4-3949 in the G347.3-0.5 SNR, the first ever performed for this source, with the NTT and the VLT. We detected a few objects close to the derived Chandra X-ray error circle. However, if they are stars, the association with the CCO would not be compatible with its current values of distance and hydrogen column density. Similar to the cases of the CCOs in PKS 1209-51, Puppis A (Wang et al. 2007), Cas A (Fesen et al. 2006) and RCW 103 (De Luca et al. 2008), our results argue against the presence of a companion star, unless it is later than M-type, and favour the INS scenario. The identification of the faintest candidate with the neutron star itself cannot be firmly excluded, while the identification with a fallback disk is ruled out by its non-detection in the $I$ band. Thus, we conclude that the 1WGA J1713.4-3949 counterpart is still unidentified. Deeper optical/IR observations are needed to pinpoint new candidates. Although the source is apparently steady in X-rays, flux variations as observed in the RCW 103 CCO (Gotthelf et al. 1999) cannot be a priory excluded. A prompt IR follow-up would then increase the chances of identifying the 1WGA J1713.4-3949 counterpart.

Acknowledgements. R.P.M. warmly thanks N. Ageorges (ESO) for her friendly support at the telescope, D. Dobrzycka (ESO) for reducing the IR data with the NACO pipeline. R.P.M. acknowledges STFC for support through a rolling grant.

\section{References}

Cassam-Chenaï, G., Decourchelle, A., Ballet, J., et al. 2004, A\&A, 427, 199 De Luca, A. 2008, in Proc. of 40 Years of Pulsars: Millisecond Pulsars, Magnetars and More, AIP, 938, 311

De Luca, A., Caraveo, P. A., Mereghetti, S., Tiengo, A., \& Bignami, G. F. 2006, Science, 313,814

De Luca, A., Mignani, R. P., Zaggia, S., et al. 2008, ApJ, in press [arXiv:0803.2885]

Ducati, J. R., Bevilacqua, C. M., Rembold, S. B., \& Ribeiro, D. 2001, ApJ, 558, 309

Fesen, R. A., Pavlov, G. G., \& Sanwal, D. 2006, ApJ, 636, 848

Gotthelf, E. V., \& Halpern, J. P. 2007, ApJ, 664, L35

Gotthelf, E. V., Petre, R., \& Vasisht, G. 1999, ApJ, 514, L107

Halpen, J. P., Gotthelf, E. V., Camilo, F., \& Seward, F. D. 2007, ApJ, 665, 1304

Høg, E., Fabricius, C., Makarov, V. V., et al. 2000, A\&A, 355, L27

Krause, O., Rieke, G. H., Birkmann, S. M., et al. 2005, Science, 308, 1064

Landolt, A. U. 1983, AJ, 88, 439

Lazendic, J. S., Slane, P. O., Gaensler, B. M., Plucinsky, P. P., \& Hughes, J. P. 2003, ApJ, 593, L27

Lazendic, J. S., Slane, P. O., Gaensler, B. M., et al. 2004, ApJ, 602, 271

Mignani, R. P., De Luca, A., Zaggia, S., et al. 2007a, A\&A, 473, 833

Mignani, R. P., Perna, R., Rea, N., et al. 2007b, A\&A, 471, 265

Pavlov, G. G., Zavlin, V. E., Aschenbach, B., Trümper, J., \& Sanwal, D. 2000, ApJ, 531, L35

Pavlov, G. G., Sanwal, D., Garmire, G. P., \& Zavlin, V. E. 2002, in Proc. of Neutron Stars in Supernova Remnants, ASP Conf. Ser., 271, 247

Pavlov, G. G., Sanwal, D., \& Teter, M. 2004, IAU Symp., 218, 239

Perna, R., Hernquist, L., \& Narayan, R. 2000, ApJ, 541, 344

Pfeffermann, E., \& Aschenbach, B. 1996, MPE Rep., 263, 267

Pizzolato, F., Colpi, M., De Luca, A., Mereghetti, S., \& Tiengo, A. 2008, ApJ, accepted [arXiv: 0803.1373]

Possenti, A., Cerutti, R., Colpi, M., \& Mereghetti, S. 2002, A\&A, 387, 993

Predehl, P., \& Schmitt, J. H. M. M. 1995, A\&A, 293, 889

Skrutskie, M. F., Cutri, R. M., Stiening, R., et al. 2006, AJ, 131, 1163

Slane, P., Gaensler, B. M., Dame, T. M., et al. 1999, ApJ, 525, 357

Stetson, P. B. 1992, Astronomical Data Analysis Software and Systems I, ASP Conf. Ser., 25, 297

Wang, Z., Chakrabarty, D., \& Kaplan, D. L. 2006, Nature, 440, 772

Wang, Z., Kaplan, D., \& Chakrabarty, D. 2007, ApJ, 655, 261

Zaggia, S. R., Piotto, G., \& Capaccioli, M. 1997, A\&A, 327, 1004

Zharikov, S. V., Shibanov, Yu. A., Mennickent, R. E., et al. 2004, A\&A, 417, 1017 